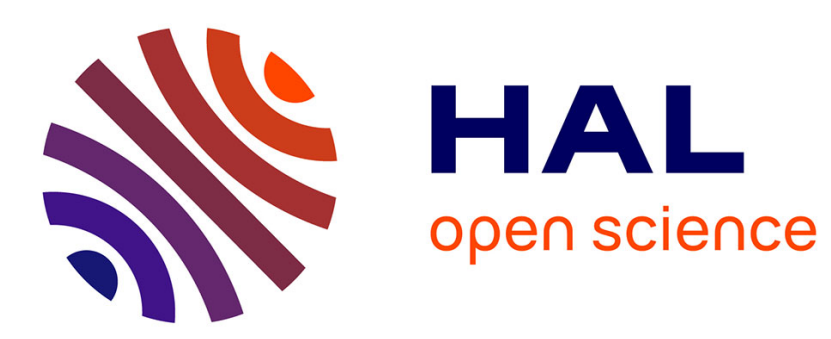

\title{
Characterization of Permanent Magnet Magnetization
}

Laure Arbenz, Olivier Chadebec, Christophe Espanet, Youness Rtimi, Gilles

Cauffet

\section{To cite this version:}

Laure Arbenz, Olivier Chadebec, Christophe Espanet, Youness Rtimi, Gilles Cauffet. Characterization of Permanent Magnet Magnetization. IEEE Transactions on Magnetics, 2017, 53 (11), pp.8109504. 10.1109/TMAG.2017.2692391 . hal-02277803

\section{HAL Id: hal-02277803 \\ https://hal.science/hal-02277803}

Submitted on 9 Nov 2020

HAL is a multi-disciplinary open access archive for the deposit and dissemination of scientific research documents, whether they are published or not. The documents may come from teaching and research institutions in France or abroad, or from public or private research centers.
L'archive ouverte pluridisciplinaire HAL, est destinée au dépôt et à la diffusion de documents scientifiques de niveau recherche, publiés ou non, émanant des établissements d'enseignement et de recherche français ou étrangers, des laboratoires publics ou privés. 


\title{
Characterization of Permanent Magnet Magnetization
}

\author{
Laure Arbenz $^{1}$, Olivier Chadebec ${ }^{2}$, Christophe Espanet ${ }^{1}$, Youness Rtimi ${ }^{1,2}$, and Gilles Cauffet ${ }^{2}$ \\ ${ }^{1}$ Research and Development Department, Moving Magnet Technologies, 25000 Besançon, France \\ ${ }^{2}$ G2Elab, CNRS, Grenoble INP, University of Grenoble Alpes, F-38000 Grenoble, France
}

The performances of electrical machines highly depend on the behavior of materials, such as steel, copper, and permanent magnet. This paper focuses on permanent magnets whose magnetization must be precisely known. Thus a non-destructive approach which includes a combination of experiments and inverse problem, using the magnetostatic moment method, is presented in order to determine the magnetization of the magnet. With a Hall sensor, a scan of the field around the sample is made. These measurements and a numerical approach allow us to deduce the sample magnetization. Studies of two samples illustrate the advantages of this method.

Index Terms - Magnetostatic moment method, measurement, permanent magnet.

\section{INTRODUCTION}

$\mathbf{M}$ ANY mechatronic systems include a permanent magnet whose magnetization must be precisely defined and known [1], [2]. For example, a fault on a magnet can drastically reduce the properties of an electric machine (through increased detent torque or iron losses...). The standard method to measure the magnetic moment of permanent magnets is the use of a Helmholtz coil in combination with a fluxmeter [3]. This approach only makes sense for a two-pole magnet, and the angle of the magnetization is quite complex to identify.

For a magnetization with more complex patterns than a mere bipolar orientation, an alternative approach must be applied. It is possible to measure the magnetic stray field of the specimen with a magnetic field sensor, typically a Hall effect probe. The magnetic moments and the magnetization angles can then be deduced from the measured signals by using an adapted algorithm. For example, this measurement process can be applied in-line during the manufacturing process to detect bipolar magnets with unknown orientation [4].

However, this task is not trivial and leads to the resolution of an inverse problem. Starting from the stray magnetic field measured around the magnet on several points (i.e., the effect), the algorithm has to reconstruct the local distribution of magnetization (i.e., its origin or sources). This kind of identification problem is known to be ill-posed, meaning that the solution is not stable versus the measurement noise: mathematically speaking, the solution is said to be "non-unique."

Before solving an inverse problem, the first step is to solve the forward one. In our case, Maxwell equations under magnetostatic assumption have to be considered. A key point is the choice of the numerical method. This choice will determine the numerical link between the magnetization and the generated stray magnetic field. Once this relation is determined, it has to be inverted.

With the finite element (FE), which is the most classical method used for static magnetic field calculation, it is necessary to mesh the air [5]. But if the magnetic circuit is open, both the air box and the mesh must be large. Moreover, this method is known to suffer from numerical noise when calculating magnetic fields around magnets. Another drawback is that the forward relation between magnetization and stray field in air is not explicit.

On the other hand, integral methods offer an explicit and direct link between the magnetization and the field in the surrounding air region. Moreover, this region does not have to be meshed, and integral approaches are well known to be very accurate if focused on the computation of stray fields. This is why it is suitable and efficient to use integral methods in the context of magnetostatic inverse problems.

A few papers have already been published on that topic. In [6], an integral inverse formulation similar to the one used in this paper is proposed, but it is limited to 2-D cases. In [7] and [8], the approach is improved, also covering ferromagnetic materials (with high permeability), but remains limited to magnetic sheets and thus 2-D magnetizations. In a more recent work, the determination of magnetizations of 3 -D volumes is proposed. The forward relation is based on a coupling between FE and surface integral methods [9]. The uniqueness of the solution is ensured with an optimization process, coupled with a regularization function. In this paper, 3-D magnetization distribution is also identified, but the use of linear purely integral formulation is preferred. The uniqueness of the solution is then implemented by truncating the matrix spectrum.

The paper is structured as follows. First, the inverse problem formulation is defined; then, the experimental setup is described, and finally, the method is used for the characterization of different samples.

\section{INVERSE PROBLEM FORMULATION}

\section{A. Forward Integral Formulation}

Let us consider a ferromagnetic domain $\Omega$ associated with an unknown distribution of magnetization. This magnetization creates an external flux density which can be expressed by the following integral equation [10]:

$$
\boldsymbol{B}(P)=-\frac{\mu_{0}}{4 \pi} \nabla \quad \frac{\boldsymbol{M} \cdot \boldsymbol{r}}{r^{3}} d \Omega
$$


where $\boldsymbol{B}$ is the flux density (T), $\mathrm{P}$ is a point located in the surrounding air region, $\boldsymbol{M}$ is the magnetization $(\mathrm{A} / \mathrm{m})$, and $\boldsymbol{r}$ is a vector linking the integration point in $\Omega$ to point $\mathrm{P}$. This expression has the advantage of explicitly linking the source which has to be determined (i.e., the magnetization) to its effect (i.e., the flux density in the surrounding air region).

\section{B. Integral Computation}

Let us mesh the domain $\Omega$ into $n$ volume elements. We assume that a uniform magnetization is associated with each of them. Integral (1) presents a $\left(\sim 1 / \mathrm{r}^{3}\right)$ singularity. Computing it directly by numerical integration can lead to significant inaccuracies, especially if the flux density has to be expressed in close proximity to the magnetized material. An alternative is using the well-known equivalent magnetic charge distribution based on Coulombian approach [10]. The magnetization $\boldsymbol{M}$ is constant for each element, so its divergence vanishes such as no volume magnetic charge exists. Thus, the field is created only by surface charge distributions $\boldsymbol{M} \cdot \boldsymbol{n}$, located on the surfaces delimiting volume elements, with vector $\boldsymbol{n}$ being the external normal of volume elements. The advantage of this approach is to present a reduced singularity $\left(\sim 1 / \mathrm{r}^{2}\right)$ as compared to $(1)\left(\sim 1 / \mathrm{r}^{3}\right)$. Thus, we get [7], [8]

$$
\boldsymbol{B}(P)=-\frac{\mu_{0}}{4 \pi} \sum_{i}^{n} \sum_{i}^{n_{f}} \int_{S_{j}} \boldsymbol{M}_{\boldsymbol{i}} \cdot \boldsymbol{n}_{\boldsymbol{j}} \frac{\boldsymbol{r}}{r^{3}} d S_{j}
$$

where $S_{j}$ is the $j$ th face of element $i, \boldsymbol{n}_{\boldsymbol{j}}$ is the external normal vector, and $\boldsymbol{n}_{\boldsymbol{f}}$ is the number of faces associated with one element of the mesh. The integral can be very accurately computed using well-known analytical expressions, even if $r$ is very small (i.e., when sensors are located very close to the ferromagnetic material). Equation (2) can be interpreted as the linear relation linking the magnetization to the flux density measured by one sensor.

\section{Linear Inverse Problem Formulation}

Let us consider that $m$ field probes are located in the "air" region surrounding the magnetic structure. In addition, a cartesian vector basis is associated with each volume element, leading to three degrees of freedom for each unknown magnetization. Thus, we get the following linear matrix system:

$$
B_{\text {mes }}=[A] M
$$

where $\boldsymbol{B}_{\text {mes }}$ is the $3 \times m$ measurement vector, $[\boldsymbol{A}]$ a $(3 \times n$, $3 \times m$ ) matrix, and $\boldsymbol{M}$ represents $3 \times n$ vectors of the unknowns.

This inverse problem is classically ill-posed [11], and its solution is not unique. In fact, even a very low noise on magnetic measurements can lead to huge variations of the solution. If so, the resolution process of the inverse problem needs to be stabilized. Many different techniques exist, but a good strategy to solve (3) is to use single value decomposition to compute the pseudoinverse of $[\boldsymbol{A}]$ and to truncate its spectrum in order to limit the noise amplification

$$
\boldsymbol{M}=[\boldsymbol{A}]^{\dagger} \boldsymbol{B}_{\text {mes. }} \text {. }
$$

A discrete evaluation of the magnetization is then obtained for the defined mesh. (a)

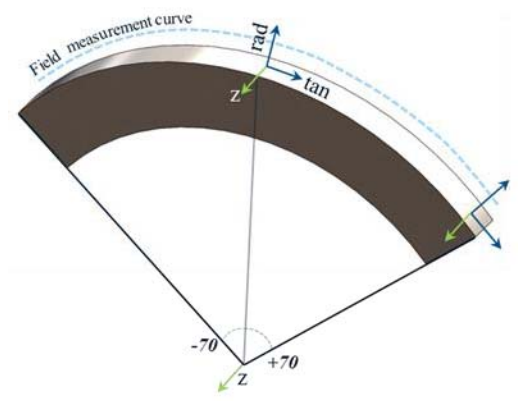

(b)

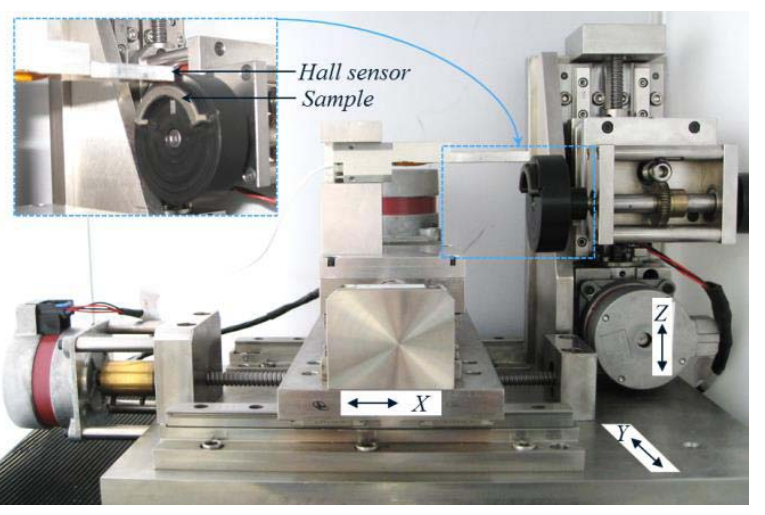

Fig. 1. (a) Shape sample. (b) Experimental setup of the proposed method.

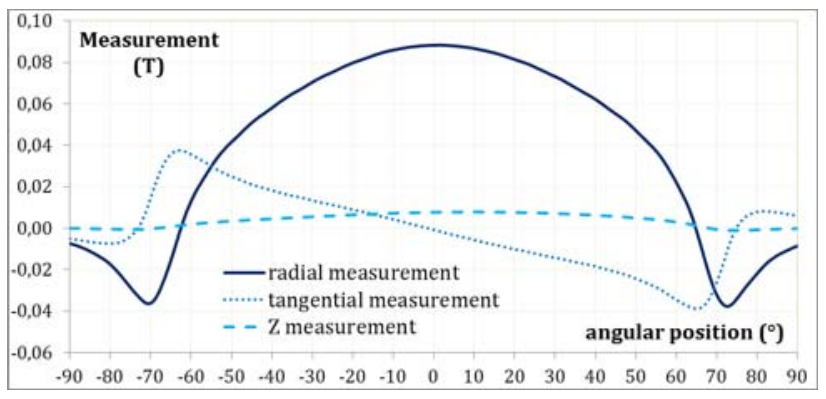

Fig. 2. Measured field (magnetic flux density) versus angular position.

\section{EXPERIMENTAl Setup AND Test Procedure}

To measure the magnetic stray field of a given specimen presented in Fig. 1(a), a three-axes Hall effect sensor is used (Senis, model F3A-03C02C-A.2T2K5J-E). The full scale of this sensor is $\pm 0.2 \mathrm{~T}$ for a total measurement accuracy below $\pm 0.1 \%$. A sensitivity study shows that the relative position between this sensor and the magnet must be accurately known. For this purpose, a homemade precision positioning bench is used [Fig. 1(b)]. The Hall sensor is fixed on an aluminum fixture and a non-magnetic support made of Ertacetal (POM plastics) tailored to fit the magnet geometry is fixed to the positioning bench. These non-magnetic surroundings of the magnet minimize potential risks of disturbing the measurement. The control of the positioning bench and the acquisition of the data are completely automated, effectively mitigating operator influences on the measurement process and thus its results.

For samples with an annular (or circular segment) shape [Fig. 1(a)], the sensor is fixed in position, while the specimen is rotated. With this experimental protocol, it is possible to measure the field on equidistant curves (arcs) surrounding the sample (Fig. 2). This operation is repeated for 11 different 


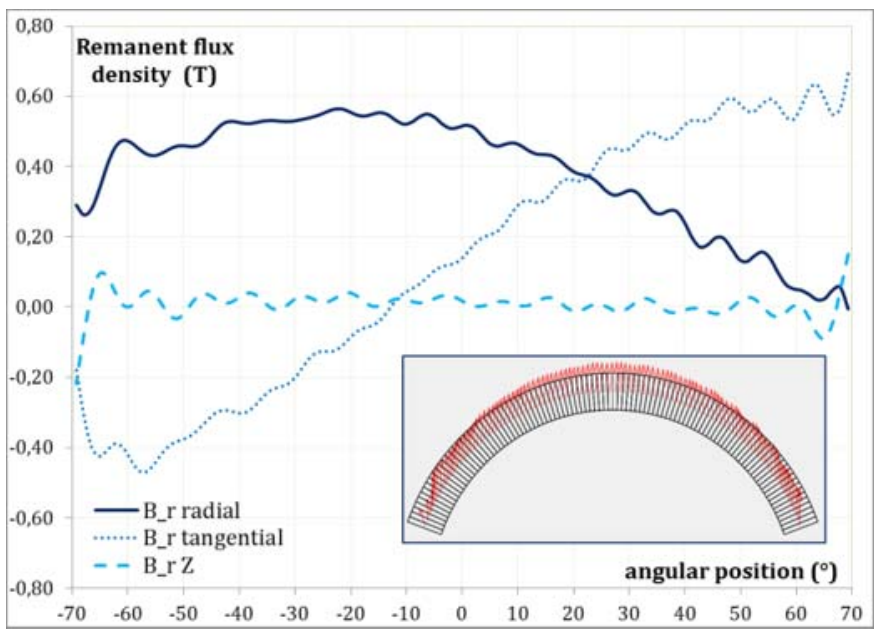

Fig. 3. Magnetization of the magnet deduced by the method. Overlay: visualization of magnetization vectors.

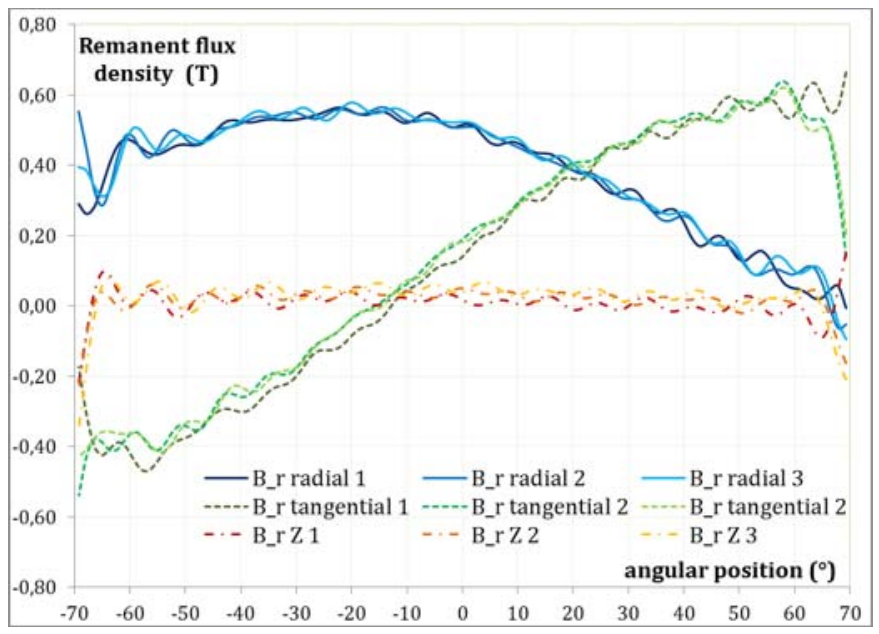

Fig. 4. Magnetization of the magnet deduced by the method (three measurements).

curves around the sample, which allows us to properly map the surrounding field.

\section{RESUlts AND Discussion}

\section{A. Deduced Magnetization}

The magnetization of the sample can be deduced from the field measurement with a numerical method. The chosen method is the magnetostatic moments method (magnetostatic MoM) [8], [12]. As explained in Section I, the MoM is more powerful than the classical FE because surrounding air does not require any meshing and especially since MoM can easily be inverted. This method is thus well adapted to identify magnetization patterns. In this paper, numerical problems are solved with a software package using the magnetic moment method and called LOCAPI. Results of the sample presented in Fig. 1(a) are shown in Fig. 3. We can conclude that the sample has a diametrical magnetization.

\section{B. Uniqueness of Solution}

It is important to determine whether the calculated magnetization is the unique solution of the problem. To check this, the

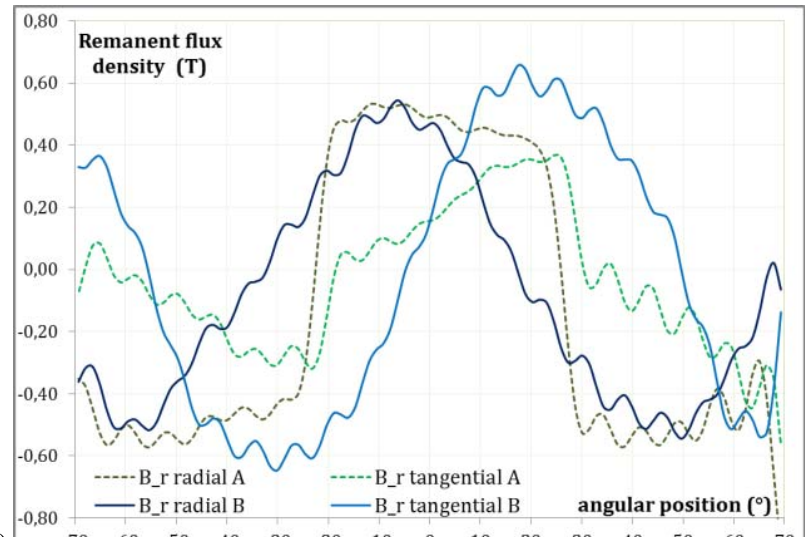

(a)
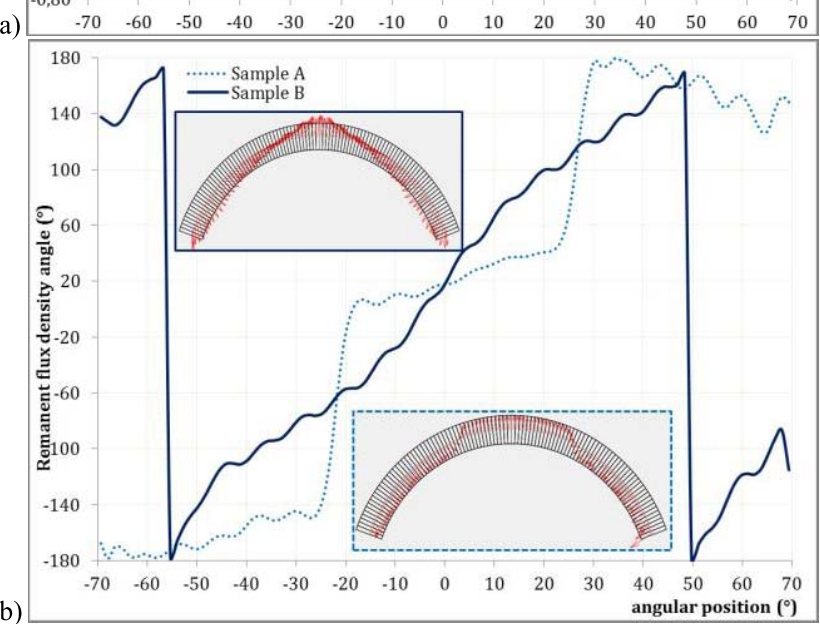

Fig. 5. (a) Radial and tangential magnetization of samples A and B. (b) Remanent flux density angle versus angular position.

non-observable space magnetization must be determined [13]. The solution vector $\boldsymbol{M}$ is projected onto the null space of the matrix $\boldsymbol{A}$ using the adapted projection operator

$$
\boldsymbol{M}_{\mathrm{no}}=\left(\boldsymbol{I}-[\boldsymbol{A}]^{\dagger} \boldsymbol{A}\right) \boldsymbol{M} .
$$

If $\boldsymbol{M}_{\mathbf{n o}}$ is sufficiently low compared to $\boldsymbol{M}$, the solution can be considered as unique. From a physical point of view, the component $\boldsymbol{M}_{\mathbf{n o}}$ which satisfies $\boldsymbol{A} \cdot \boldsymbol{M}_{\mathbf{n o}}=0$ is a magnetization which does not leave any traces on the sensors. Thus, $\boldsymbol{M}_{\mathbf{n o}}$ is the non-returnable information using the inversion procedure.

It is interesting to note that $\boldsymbol{A}$ is only a function of the geometry of the problem (sample geometry and mesh, location of the measurements). Therefore, the only way to overcome this issue is changing the sensors' spatial positions or the sample mesh. Mathematically, this equals reducing the nonobservable space (i.e., null space of $\boldsymbol{A}$ ). It is thus possible to determine whether the measurement definition and the mesh are properly adapted before even launching the test sequence.

\section{Sensitivity Study}

To illustrate the reproducibility of the method, the same sample has been characterized three times, using the same experimental and numerical methods. These three measurement runs are completely independent of each other. The magnetization results are given in Fig. 4. 
The standard deviation is less than $0.04 \mathrm{~T}(0.06 \mathrm{~T})$ for radial (tangential) remanent flux density on an angular aperture of $\pm 60^{\circ}$ (the angular aperture of the magnet is $\pm 70^{\circ}$ ). So the reproducibility of the method is sufficient to clearly identify the magnetization of the magnet.

However, the standard deviation is five times larger at the tips of the magnet $\left(-70^{\circ}\right.$ to $-60^{\circ}$ and $+60^{\circ}$ to $\left.+70^{\circ}\right)$. This phenomenon is due to a difference between the physical magnet position during the measurements and the theoretical magnet position used in the numerical model. The magnetization at the end of the magnet is therefore either oversized or undersized.

\section{Application of Measurement Method}

We then propose to study two samples A and B with the same physical shape [Fig. 1(a)] but different magnetization patterns. Both samples are what we call "three-pole magnets" (e.g., a N / S / N pattern), one of which has a radial magnetization and the other one a sinusoidal magnetization. The radial and tangential magnetizations are given in Fig. 5(a). Both samples are clearly different, but it is simpler to evaluate when comparing the flux density versus the angular position [Fig. 5(b)]. It is evident that sample A is radial (visible steps at the pole transitions) and sample B is sinusoidal (linear flux rotation).

\section{CONCLUSION}

The proposed approach allows deducing 3-D magnetization distribution of a magnet from stray magnetic field measurements. It is based on the inversion of a pure volume integral formulation of the magnetic field regularized with a matrix spectrum truncation. Being a non-destructive approach, it involves a combination of experiments and inverse problem using the magnetostatic moment method. The procedure was applied to samples with identical geometry but different magnetization patterns. The results allow us to reliably distinguish samples without any ambiguity. A major advantage of the method is that it can be applied whatever the geometry of the sample. Further improvement may be possible by adding a probe positioning sensor on the test fixture. This should substantially increase the reproducibility of the method. Moreover, it may make sense to find a way to automate the localization of measurement points, depending on geometry and mesh of the sample to be investigated.

\section{REFERENCES}

[1] G. Loussert, "An efficient and optimal moving magnet actuator for active vibration control," in Proc. Actuator, Bremen, Germany, 2016, paper A6.2.

[2] A. Gilson, S. Tavernier, F. Dubas, D. Depernet, and C. Espanet, "2-D analytical subdomain model for high-speed permanent-magnet machines," in Proc. ICEMS, 2015, pp. 1508-1514.

[3] Magnetic Materials-Part 14: Methods of Measurement of the Magnetic Dipole Moment of a Ferromagnetic Material Specimen by the Withdrawal or Rotation Method, International Standard IEC 60404-14, 2002.

[4] A. Meyer et al., "Concept for magnet intra logistics and assembly supporting the improvement of running characteristics of permanent magnet synchronous motors," Procedia CIRP, vol. 43, pp. 356-361, Sep. 2016.

[5] L. Arbenz, A. Benabou, S. Clénet, J.-C. Mipo, and P. Faverolle, "Characterization of the local incremental permeability of a ferromagnetic plate based on a four needles technique," IEEE Trans. Magn., vol. 53, no. 3, Mar. 2017, Art. no. 6000207.

[6] H. Igarashi, T. Honma, and A. Kost, "Inverse inference of magnetization distribution in cylindrical permanent magnets," IEEE Trans. Magn., vol. 36, no. 4, pp. 1168-1171, Apr. 2000.

[7] O. Chadebec, J. L. Coulomb, J. P. Bongiraud, G. Cauffet, and P. L. Thiec, "Recent improvements for solving inverse magnetostatic problem applied to thin shells," IEEE Trans. Magn., vol. 38, no. 2, pp. 1005-1008, Mar. 2002.

[8] O. Chadebec, J.-L. Coulomb, and F. Janet, "A review of magnetostatic moment method," IEEE Trans. Magn., vol. 42, no. 4, pp. 515-520, Apr. 2006

[9] F. Bruckner et al. (Aug. 2016). "Solving large-scale inverse magnetostatic problems using the adjoint method." [Online]. Available: https://arxiv.org/abs/1609.00060

[10] E. Durand, "Magnétostatique," Masson et Cie, Paris, France, 1968.

[11] P. C. Hansen and D. P. O'leary, "The use of the L-curve in the regularization of discretization of discrete ill-posed problem," SIAM J. Sci. Comput., vol. 14, pp. 1487-1503, Apr. 1993.

[12] R. F. Harrington, Field Computation by Moment Methods. New York, NY, USA: IEEE Press, 1993.

[13] R. Penrose and J. A. Todd, "A generalized inverse for matrices," Math. Proc. Cambridge Philos. Soc., vol. 51, no. 3, p. 406, 1955. 